\title{
ON THE EXISTENCE OF MULTIPLE POSITIVE ENTIRE SOLUTIONS FOR A CLASS OF QUASILINEAR ELLIPTIC EQUATIONS
}

\author{
YANG ZUODONG
}

Received 21 June 2005; Revised 22 November 2005; Accepted 22 January 2006

Our goal is to establish the theorems of existence and multiple of positive entire solutions for a class quasilinear elliptic equations in $\mathbb{R}^{N}$ with the Schauder-Tychonoff fixed point theorem as the principal tool. In many articles, the theorems of existence and multiple of positive entire solutions for a class semilinear elliptic equations are established. The results of the semilinear equations are extended to the quasilinear ones and the results of semilinear equations are developed.

Copyright (c) 2006 Hindawi Publishing Corporation. All rights reserved.

\section{Introduction}

In this paper, we consider the existence of multiple positive entire solutions for a class of quasilinear elliptic equation

$$
\operatorname{div}\left(|\nabla u|^{p-2} \nabla u\right)=f(x, u, \nabla u), \quad x \in \mathbb{R}^{N},
$$

where $p>1$.

Equations of the above form are mathematical models occurring in the studies of the $p$-Laplace equation, generalized reaction-diffusion theory, non-Newtonian fluid theory [7], and the turbulent flow of a gas in porous medium [2]. In the non-Newtonian fluid theory, the quantity $p$ is characteristic of the medium. Media with $p>2$ are called dilatant fluids and those with $p<2$ are called pseudoplastics. If $p=2$, they are Newtonian fluids.

By a positive entire solution of (1.1) we mean a function $u \in W^{1, p}\left(\mathbb{R}^{N}\right) \cap C^{1}\left(\mathbb{R}^{N}\right)$ which satisfies (1.1) at every point of $\mathbb{R}^{N}$ in a weak sense with $u>0$ in $\mathbb{R}^{N}$ (see [4] and references therein), that is $u \in W^{1, p}\left(\mathbb{R}^{N}\right) \cap C^{1}\left(\mathbb{R}^{N}\right)$ which satisfies

$$
-\int_{\mathbb{R}^{N}}|\nabla u|^{p-2} \nabla u \cdot \nabla \psi d x=\int_{\mathbb{R}^{N}} f(x, u, \nabla u) \psi d x \quad \forall \psi \in C_{0}^{\infty}\left(\mathbb{R}^{N}\right)
$$

and $u>0$ in $\mathbb{R}^{N}$. 
2 Multiple entire solutions for quasilinear elliptic equations

The existence and nonexistence of entire solutions, existence of multiple positive entire solutions of (1.1) for $f(x, u, \nabla u)=q(x) f(u)$ or $f(x, u, \nabla u)=-f(x, u)$, have been studied in previous papers (see $[22,24,25])$. Some other problems have also been treated by many other authors. See, for example, [5, 6, 8, 13-15, 18, 23, 26, 27].

When $f:(0, \infty) \rightarrow(0, \infty)$ and $q: \mathbb{R}^{N} \rightarrow(0, \infty)$ are continuous functions, and

$$
\int_{1}^{\infty}\left(\int_{0}^{u} f(s) d s\right)^{-1 / p} d u=\infty
$$

it has been shown in [22] that there exist entire radially symmetric solutions of the problem

$$
\operatorname{div}\left(|\nabla u|^{p-2} \nabla u\right)=q(x) f(u), \quad x \in \mathbb{R}^{N}
$$

On the other hand, it was shown in [24] that the problem

$$
\operatorname{div}\left(|\nabla u|^{p-2} \nabla u\right)+f(x, u)=0, \quad x \in \mathbb{R}^{N},
$$

possesses infinitely many positive entire solutions. When $f(x, u)$ defined on $\mathbb{R}^{N}$ is locally Hölder continuous in $x$ and is locally Lipschitz continuous in $u$; there exist a locally Hölder continuous function $\psi(r) \geq 0$ on $[0, \infty), \int_{0}^{\infty}\left(\int_{0}^{s} \psi(t) d t\right)^{1 /(p-1)} d s<\infty$, and a locally Lipschitz continuous function $F(u)>0$ on $(0, \infty)$ such that

$$
f(x, u) \leq \psi(|x|) F(u), \quad(x, u) \in \mathbb{R}^{N} \times(0, \infty),
$$

and $\lim _{u \rightarrow 0}\left(F(u) / u^{p-1}\right)=0$.

Moreover, it was also shown in [25] that the problem

$$
\operatorname{div}\left(|\nabla u|^{p-2} \nabla u\right)+q(x) u^{-\gamma}=0, \quad x \in \mathbb{R}^{N},
$$

has a positive entire solution if $1<p<N, 0 \leq \gamma<p-1$, and $q(x) \in C\left(\mathbb{R}^{+}\right)$satisfy

$\left(\mathrm{A}_{1}\right)$ wherever $q\left(x_{0}\right)=0, \exists r>0$ such that $q(x)>0$ on $\partial B\left(x_{0}, r\right)$, where $B\left(x_{0}, r\right)$ is the ball of radius $r$ centered at $x_{0}$;

$\left(\mathrm{A}_{2}\right)$ for any $0<\varepsilon<(N-p)(p-1-|\gamma|) /(p-1)$,

$$
\int_{1}^{\infty} r^{p+\mathcal{E}-1+[(N-p)|\gamma| /(p-1)]} m(r) d r<\infty ;
$$

$\left(\mathrm{A}_{3}\right)$ for $r \in(0,1)$,

$$
q(r)=O\left(r^{-\delta}\right), \quad \delta<1
$$

Motivated by the results of the above-cited papers, we further study the existence of multiple positive entire solutions for (1.1), the results of the semilinear equations are extended to the quasilinear ones. We can find the related results for $p=2$ in $[10,11,17$, $20,21]$. The main differences between $p=2$ and $p \neq 2$ are known in $[5,6]$. When $p=2$, 
it is well known that all positive solutions in $C^{2}\left(B_{R}\right)$ of the problem

$$
\begin{gathered}
\triangle u+f(u)=0 \quad \text { in } B_{R}, \\
u(x)=0 \quad \text { on } \partial B_{R}
\end{gathered}
$$

are radially symmetric solutions for very general $f$ (see [3]). Unfortunately, this result does not apply to the case $p \neq 2$. Kichenassamy and Smoller showed that there exist many positive nonradial solutions of the above problem for some $f$ (see [9]). The major stumbling block in the case of $p \neq 2$ is that certain nice features inherent to the case $p=2$ seem to be lost or at least difficult to verify. In this paper, we obtain the existence of multiple positive entire solutions for a class of $f$, extended to the results in $[11,20,21]$ and complement the results by $[22,24,25]$.

\section{Some preliminary lemmas}

Before we prove the main results, we need the following definitions and lemmas.

Definition 2.1. $\bar{u} \in W^{1, p}\left(\mathbb{R}^{N}\right) \cap C^{1}\left(\mathbb{R}^{N}\right)$ is called a supersolution to problem (1.1) if

$$
-\int_{\mathbb{R}^{N}}|\nabla \bar{u}|^{p-2} \nabla \bar{u} \cdot \nabla \psi d x \geq \int_{\mathbb{R}^{N}} f(x, \bar{u}, \nabla \bar{u}) \psi d x \quad \forall \psi \in C_{0}^{\infty}\left(\mathbb{R}^{N}\right)
$$

and $\bar{u}>0$ in $\mathbb{R}^{N}$. Similarly, $\underline{u} \in W^{1, p}\left(\mathbb{R}^{N}\right) \cap C^{1}\left(\mathbb{R}^{N}\right)$ is called a subsolution to problem (1.1) if

$$
-\int_{\mathbb{R}^{N}}|\nabla \underline{u}|^{p-2} \nabla \underline{u} \cdot \nabla \psi d x \leq \int_{\mathbb{R}^{N}} f(x, \underline{u}, \nabla \underline{u}) \psi d x \quad \forall \psi \in C_{0}^{\infty}\left(\mathbb{R}^{N}\right)
$$

and $\underline{u}>0$ in $\mathbb{R}^{N}$.

For (1.1), the following hypotheses on $f$ are adopted.

(A) $f(x, u, v)$ is a continuous function in $\mathbb{R}^{N} \times \mathbb{R}^{+} \times \mathbb{R}^{N}$ and locally Lipschitz continuous.

(B) For every bounded domain $\Omega \subset \mathbb{R}^{N}$, for any $M>0, \exists \rho(\Omega, M)>0$ such that

$$
|f(x, u, v)|<\rho(\Omega, M)\left(1+|v|^{p}\right), \quad x \in \Omega, 0 \leq u \leq M, v \in \mathbb{R}^{N} .
$$

(C) There exist nonnegative continuous functions defined in $\left(\mathbb{R}^{+}\right)^{3}, F_{1}(r, u, v)$, and $F_{2}(r, u, v)$, which are local Lipschitz continuous and satisfy

$$
F_{1}(|x|, u,|v|) \leq f(x, u, v) \leq F_{2}(|x|, u,|v|)
$$

where $(x, u, v) \in \mathbb{R}^{N} \times \mathbb{R}^{+} \times \mathbb{R}^{N},|x|=\left(\sum_{i=1}^{n} x_{i}^{2}\right)^{1 / 2}$.

LemmA 2.2. Let $\bar{u}, \underline{u} \in W^{1, p}\left(\mathbb{R}^{N}\right) \cap C^{1}\left(\mathbb{R}^{N}\right)$, respectively, be supersolution and subsolution of (1.1) on $\mathbb{R}^{N}$ with $\underline{u}(x) \leq \bar{u}(x)$ on $\mathbb{R}^{N}$, and let $(A),(B)$ hold. Then, (1.1) possesses an entire solution $u(x)$ with $\underline{u}(x) \leq u(x) \leq \bar{u}(x)$ on $\mathbb{R}^{N}$. 
4 Multiple entire solutions for quasilinear elliptic equations

Proof. Let $B_{R}$ be the ball with radius $R$ in $\mathbb{R}^{N}$. Consider the boundary value problem

$$
\begin{gathered}
\operatorname{div}\left(|\nabla u|^{p-2} \nabla u\right)=f(x, u, \nabla u), \quad x \in B_{R}, \\
\left.u\right|_{\partial B_{R}}=g,
\end{gathered}
$$

where $g(x)$ is a function that satisfies $\underline{u}(x) \leq g(x) \leq \bar{u}(x)$. For (2.5), (2.6), $\bar{u}(x)$ is still a supersolution (for every $R$ ) and $\underline{u}(x)$ is still a subsolution (for every $R$ ), and $\bar{u}(x) \geq \underline{u}(x)$ in $B_{R}$. By $C^{1, \alpha}\left(\bar{B}_{R}\right)$ estimates in [13] and monotonic iteration [16] or [1,8], one concludes that there exists $u \in C^{1}\left(\bar{B}_{R}\right)$, which is a weak solution of (1.1) with $\underline{u}(x) \leq u_{R}(x) \leq \bar{u}(x)$ in $B_{R}$.

Now, we want to apply elliptic interior estimates together with a diagonal process to conclude that $\left\{u_{R}: R \geq 1\right\}$ has a subsequence $\left\{u_{R_{k}}: R_{k} \uparrow \infty\right\}$ such that $\left\{u_{R_{k}}\right\}$ converges to a function $u$ in $\mathbb{R}^{N}$ (pointwise) and this convergence is in $C^{1}$ on every compact set in $\mathbb{R}^{N}$. (Therefore, $u \in C^{1}$ and $\operatorname{div}\left(|\nabla u|^{p-2} \nabla u\right)=f(x, u, \nabla u)$ with $\underline{u}(x) \leq u(x) \leq \bar{u}(x)$, and this concludes the proof.)

Step 1 . On $B_{2},\left\{u_{R}: R \geq 2\right\}$ is uniformly bounded by $\bar{u}(x)$ and $\underline{u}(x)$. Since both $\bar{u}(x)$ and $\underline{u}(x)$ are bounded functions on $B_{2}$, there exists $M>0$ such that $\left\|u_{R}(x)\right\|_{L^{\infty}\left(B_{2}\right)} \leq M$ for all $R \geq 2$.

From (1.1), $u_{R}$ satisfies

$$
\int_{B_{2}}\left|\nabla u_{R}\right|^{p}=-\int_{B_{2}} f u_{R}
$$

Therefore,

$$
\int_{B_{2}}\left|\nabla u_{R}\right|^{p} \leq M\left(\operatorname{meas} B_{2}\right)^{1 / q} C_{1}\left\|\nabla u_{R}\right\|_{p}
$$

Here $1 / q+1 / p=1$, and $C_{1}$ is the Sobolev embedding constant. So, $\left\|u_{R}\right\|_{1, p} \leq C_{2}$. When $1<p<N$, the embedding of $W_{0}^{1, p}\left(B_{2}\right)$ in $L^{N p /(N-p)}\left(B_{2}\right)$ implies that $u_{R} \in L^{N p /(N-p)}\left(B_{2}\right)$. Applying [12, Theorem 7.1, pages 286, 287], we obtain the estimate

$$
\sup \left\{\left|u_{R}\right| ; x \in B_{2}\right\} \leq C_{3} \text {, }
$$

here $C_{3}=C_{3}\left(\|f\|_{0}\right)$. If $p \geq N$, we get (2.9) from the Sobolev embedding theorem. Using $\left[12\right.$, Theorem 1.1, page 251], we see that $u_{R}$ belongs to $C^{\alpha}\left(\overline{B_{2}}\right)$ for some $0<\alpha<1$, and

$$
\left\|u_{R}\right\|_{C^{\alpha}} \leq C_{4},
$$

here $C_{4}$ is determined by $C_{3}$. By [19, Proposition 3.7, page 806] we also know that $u_{R}$ belongs to $C^{1, \alpha}\left(\overline{B_{2}}\right)$ and

$$
\left\|u_{R}\right\|_{C^{1, \alpha}} \leq C_{5}
$$

here $C_{5}$ is determined by $C_{4}$.

From the arguments above we see that there exists $C>0$ such that

$$
\left\|u_{R}\right\|_{C^{1+\alpha}\left(B_{1}\right)} \leq C \quad \forall R \geq 2 .
$$


Since the embedding $C^{1+\alpha}\left(B_{1}\right) \rightarrow C^{1}\left(B_{1}\right)$ is compact, there exists a sequence denoted by $\left\{u_{R_{1 j}}\right\}_{j=1, \ldots}$ (where $R_{1 j} \uparrow \infty$ ), which converges in $C^{1}\left(B_{1}\right)$. Let $u_{1}(x)=\lim _{j \rightarrow \infty} u_{R_{1 j}}(x)$ for $x \in B_{1}$; then $u_{1}$ is a solution of $(2.5)$ with $\underline{u}(x) \leq u_{1} \leq \underline{u}(x)$.

Step 2. Repeat Step 1 up to the existence of the sequence $\left\{u_{R_{1 j}}\right\}_{j=1, \ldots}$ to get a subsequence $\left\{u_{R_{2 m}}\right\}_{m=1,2, \ldots}$ converging in $C^{1}\left(B_{2}\right)$ to a limit $u_{2}$. Then likewise $u_{2}$ is a solution of (2.5), (2.6) and $\left.u_{2}\right|_{B_{1}}=u_{1}$. Repeat Step 1 again on $B_{3}, \ldots$, and so forth. In this way, we obtain a sequence $\left\{u_{R_{k j}}\right\}_{j=1,2, \ldots}$ which converges in $C^{1}\left(B_{k}\right)$ and is a subsequence of $\left\{u_{R_{(k-1) j}}\right\}$. Let $u_{k}=\lim _{j \rightarrow \infty} u_{R_{k j}}$, then $u_{k}$ is a solution of (2.5), (2.6) in $B_{k}$ and $\left.u_{k}\right|_{B_{k-1}}=u_{k-1}$.

Step 3. By a diagonal process, $\left\{u_{R_{m m}}\right\}_{m=1,2, \ldots}$ is a subsequence of $\left\{u_{R_{k j}}\right\}_{j=1,2, \ldots}$ for every $k$. Thus, on $B_{k}$ for each $k$ we have

$$
\lim _{m \rightarrow \infty} u_{R_{m m}}=u_{k}
$$

So, if we define $u(x)=\lim _{m \rightarrow \infty} u_{R_{m m}}(x)$, then $u(x)$ satisfies

$$
\operatorname{div}\left(|\nabla u|^{p-2} \nabla u\right)=f(x, u, \nabla u), \quad x \in \mathbb{R}^{N}
$$

and $\underline{u}(x) \leq u(x) \leq \bar{u}(x)$, since $\underline{u}(x) \leq u_{k}(x) \leq \bar{u}(x)$ for every $k$. This completes the proof of Lemma 2.2.

Lemma 2.3. (i) Let all $a, b>0$ and $p \geq 2$, then

$$
\left|a^{1 /(p-1)}-b^{1 /(p-1)}\right| \leq 2 \frac{|a-b|}{a^{(p-2) /(p-1)}+b^{(p-2) /(p-1)}} .
$$

(ii) Let all $a, b \geq 0$ and $1<p<2$, then

$$
\left|a^{1 /(p-1)}-b^{1 /(p-1)}\right| \leq 2^{1 /(p-1)}|a-b|\left(a^{(2-p) /(p-1)}+b^{(2-p) /(p-1)}\right) .
$$

\section{Main results}

In this section we give the following main results.

Theorem 3.1. Assume that $f$ satisfies $(A)-(C)$ and the following conditions hold.

(I) $F_{1}(r, u, v)$ and $F_{2}(r, u, v)$ are nonincreasing functions in $u \in \mathbb{R}^{+}$, nondecreasing in $v \in \mathbb{R}^{+}$for each $r \geq 0$.

(II) (a) For $1<p<2$ and fixed $r \in \mathbb{R}^{+}, F_{2}^{1 /(p-1)}(r, \lambda, m \lambda / p) / \lambda$ is nonincreasing for $\lambda \in$ $(0, \infty)$ and satisfies that

$$
\lim _{\lambda \rightarrow+\infty} \frac{F_{2}^{1 /(p-1)}(r, \lambda, m \lambda / p)}{\lambda}=0
$$

where $m=((2-p) /(N-p+1))^{(2-p) /(p-1)}$. 
6 Multiple entire solutions for quasilinear elliptic equations

(b) For $2 \leq p \leq N+1$ and fixed $r \in \mathbb{R}^{+}, F_{2}^{p-1}\left(r, \lambda, m_{1} \lambda / p\right) / \lambda$ is nonincreasing for $\lambda \in(0, \infty)$ and satisfies that

$$
\lim _{\lambda \rightarrow+\infty} \frac{F_{2}^{p-1}\left(r, \lambda, m_{1} \lambda / p\right)}{\lambda}=0,
$$

where $m_{1}=((p-2) /(N(p-1)-1))^{(p-2) /(p-1)^{2}}$.

(III) (a) For $1<p<2$, there exists a positive constant $c>0$ such that

$$
\int_{0}^{\infty} K(s) F_{2}^{1 /(p-1)}\left(s, c, \frac{m c}{p}\right) d s<\infty,
$$

where

$$
K(s)= \begin{cases}1 & \text { if } 0 \leq s<1 \\ s^{1 /(p-1)} & \text { if } s>1 .\end{cases}
$$

(b) For $2 \leq p \leq N+1$, there exists a positive constant $c$ such that

$$
\int_{0}^{\infty} K(s) F_{2}^{p-1}\left(s, c, \frac{m_{1} c}{p}\right) d s<\infty,
$$

where

$$
K(s)= \begin{cases}1 & \text { if } 0 \leq s<1 \\ s^{p-1} & \text { if } s>1\end{cases}
$$

then (1.1) has infinitely many positive entire solutions $u(x)$.

Proof. It is easy to see that under conditions (A)-(C) a positive solution $\bar{u}(x)$ of the equation

$$
\operatorname{div}\left(|\nabla \bar{u}|^{p-2} \nabla \bar{u}\right)=F_{1}(|x|, \bar{u},|\nabla \bar{u}|), \quad x \in \mathbb{R}^{N},
$$

is a supersolution of $(1.1)$ in $\mathbb{R}^{N}$; and a positive solution $\underline{u}$ of the equation

$$
\operatorname{div}\left(|\nabla \underline{u}|^{p-2} \nabla \underline{u}\right)=F_{2}(|x|, \underline{u},|\nabla \underline{u}|), \quad x \in \mathbb{R}^{N},
$$

is a subsolution of (1.1) in $\mathbb{R}^{N}$. Therefore, we only prove that (3.7), (3.8) have solutions and satisfy $\underline{u}(x) \leq \bar{u}(x)$.

We first consider (3.7), in view of the spherical symmetry of $F_{1}(|x|, v,|\nabla v|)$, it is natural to seek spherically symmetric solutions of (3.7), and thus we are led to the onedimensional initial value problems

$$
\begin{gathered}
\left(\Phi_{p}\left(y^{\prime}\right)\right)^{\prime}+\frac{N-1}{r} \Phi_{p}\left(y^{\prime}\right)=F_{1}\left(r, y,\left|y^{\prime}\right|\right), \quad r>0, \\
y(0)=\eta, \quad y^{\prime}(0)=0,
\end{gathered}
$$


where $\Phi_{p}(y)=|y|^{p-2} y$, and $\eta$ is a real number which is determined below. If $y(r)$ is a solution of $(3.9)$ on $(0, \infty)$, then $v(x)=y(|x|)$ is a supersolution of $(1.1)$ in $\mathbb{R}^{N}$.

It can be proved that the problem (3.9), (3.10) is equivalent to the following integral equation:

$$
y(r)=\eta+\int_{0}^{r}\left(\int_{0}^{s}\left(\frac{t}{s}\right)^{N-1} F_{1}\left(t, y,\left|y^{\prime}(t)\right|\right) d t\right)^{1 /(p-1)} d s
$$

Therefore we only consider integral equation (3.11). To prove that (3.11) has a solution, we consider two cases here: (i) $1<p \leq 2$ and (ii) $p>2$.

(i) For $1<p<2$, condition (II) implies that

$$
K(s) \frac{F_{2}^{1 /(p-1)}(s, \lambda, m \lambda / p)}{\lambda} \leq K(s) F_{2}^{1 /(p-1)}\left(s, c, \frac{m c}{p}\right), \quad \lambda \geq c,
$$

where $c$ is defined in (III), and for all $s \in(0, \infty)$, as $\lambda \rightarrow \infty$, we have

$$
K(s) \frac{F_{2}^{1 /(p-1)}(s, \lambda, m \lambda / p)}{\lambda} \longrightarrow 0
$$

According to (III), by Lebesgue dominated convergence theorem, we have

$$
\frac{1}{\lambda} \int_{0}^{\infty} K(s) F_{2}^{1 /(p-1)}\left(s, \lambda, \frac{m \lambda}{p}\right) d s \longrightarrow 0 \quad(\lambda \longrightarrow \infty)
$$

from (a) of (II) implying that

$$
\frac{1}{\lambda} \int_{0}^{\infty} K(s) F_{1}^{1 /(p-1)}\left(s, \lambda, \frac{m \lambda}{p}\right) d s \rightarrow 0 \quad(\lambda \longrightarrow \infty)
$$

for all $1<p<2$. From (3.15), we can choose sufficiently large constants $\eta>0$, such that

$$
\int_{0}^{\infty} K(s) F_{1}^{1 /(p-1)}\left(s, \eta, \frac{m \eta}{p}\right) d s<\frac{\eta}{p} .
$$

Let $Y$ be a set defined by

$$
Y=\left\{y \in C^{1}[0, \infty) \mid \eta \leq y(r) \leq 2 \eta, 0 \leq y^{\prime}(r) \leq \frac{m \eta}{p}\right\} .
$$

Clearly, $Y$ is a closed convex subset of $C^{1}[0, \infty)$. Furthermore, the mapping $F: Y \rightarrow$ $C^{1}[0, \infty)$ is defined by

$$
F y(r)=\eta+\int_{0}^{r}\left(\int_{0}^{s}\left(\frac{t}{s}\right)^{N-1} F_{1}\left(t, y,\left|y^{\prime}(t)\right|\right) d t\right)^{1 /(p-1)} d s, \quad r \geq 0,
$$

where the value at 0 as well as the following limit supplement define

$$
F y(0)=\lim _{r \rightarrow 0}\left[\eta+\int_{0}^{r}\left(\int_{0}^{s}\left(\frac{t}{s}\right)^{N-1} F_{1}\left(t, y,\left|y^{\prime}(t)\right|\right) d t\right)^{1 /(p-1)} d s\right]=\eta .
$$


8 Multiple entire solutions for quasilinear elliptic equations

Next we will prove step by step that $F$ is a continuous operator, mapping $Y$ into a compact subset of $Y$. The following propositions are essential.

Proposition 3.2. F is a mapping from $Y$ to $Y$.

In fact, if all $y \in Y$, it follows from (3.16) and (3.18) that

$$
\begin{aligned}
\eta \leq F y(r) & \leq \eta+\int_{0}^{r} s^{1 /(p-1)} F_{1}^{1 /(p-1)}\left(s, \eta, \frac{m \eta}{p}\right) d s \\
& \leq \eta+\int_{0}^{r} K(s) F_{1}^{1 /(p-1)}\left(s, \eta, \frac{m \eta}{p}\right) d s \leq 2 \eta
\end{aligned}
$$

and from (3.18), we have

$$
\begin{aligned}
0<(F y)^{\prime}(r) & =\left(\int_{0}^{r}\left(\frac{t}{r}\right)^{N-1} F_{1}\left(t, y,\left|y^{\prime}\right|\right) d t\right)^{1 /(p-1)} \\
& \leq\left[\left(\int_{0}^{r}\left(\frac{t}{r}\right)^{(N-1) /(2-p)} d t\right)^{2-p}\left(\int_{0}^{r} F_{1}^{1 /(p-1)}\left(t, y,\left|y^{\prime}\right|\right) d t\right)^{p-1}\right]^{1 /(p-1)} \\
& =m r^{(2-p) /(p-1)} \int_{0}^{r} F_{1}^{1 /(p-1)}\left(t, y,\left|y^{\prime}\right|\right) d t \\
& \leq m \int_{0}^{\infty} K(s) F_{1}^{1 /(p-1)}\left(s, \eta, \frac{m \eta}{p}\right) d s \leq \frac{m \eta}{p}
\end{aligned}
$$

for $r>0$. It follows from (3.21) that

$$
\lim _{r \rightarrow 0^{+}}\left[(F y)^{\prime}(r)\right]^{p-1}=\lim _{r \rightarrow 0^{+}} \frac{\int_{0}^{r} t^{N-1} F_{1}\left(t, y,\left|y^{\prime}\right|\right) d t}{r^{N-1}}=0,
$$

then $\lim _{r \rightarrow 0^{+}}(F y)^{\prime}(r)=0$. Therefore, we have

$$
(F y)^{\prime}(0)=\lim _{r \rightarrow 0^{+}} \frac{[F y(r)-F y(0)]}{r}=\lim _{\xi \rightarrow 0^{+}}(F y)^{\prime}(\xi)=0 ;
$$

and then let

$$
\left.\left(\int_{0}^{r}\left(\frac{t}{r}\right)^{N-1} F_{1}\left(t, y,\left|y^{\prime}\right|\right) d t\right)^{1 /(p-1)}\right|_{r=0}=0 .
$$

So (3.18) holds for $r \geq 0$, and we have

$$
0 \leq(F y)^{\prime}(r) \leq \frac{m \eta}{p}, \quad r \geq 0
$$


Proposition 3.3. F is continuous.

Let $\left\{y_{k}\right\}$ be a sequence in $Y$ converging to $y \in Y$. By (3.18) and Lemma 2.3, we have

$$
\begin{aligned}
& \left|F y_{k}(r)-F y(r)\right| \\
& \quad \leq\left|\int_{0}^{r}\left[\Phi_{p}^{-1}\left(\int_{0}^{s}\left(\frac{t}{s}\right)^{N-1} F_{1}\left(t, y_{k},\left|y_{k}^{\prime}\right|\right) d t\right)-\Phi_{p}^{-1}\left(\int_{0}^{s}\left(\frac{t}{s}\right)^{N-1} F_{1}\left(t, y,\left|y^{\prime}\right|\right) d t\right)\right] d s\right| \\
& \quad \leq 2^{1 /(p-1)}\left|\int_{0}^{\infty}\left(\int_{0}^{s}\left|F_{1}\left(t, y_{k},\left|y_{k}^{\prime}\right|\right)-F_{1}\left(t, y,\left|y^{\prime}\right|\right)\right| d t\right)\left(A^{(2-p) /(p-1)}+B^{(2-p) /(p-1)}\right) d s\right|,
\end{aligned}
$$

where

$$
A=\int_{0}^{s}\left(\frac{t}{s}\right)^{N-1} F_{1}\left(t, y_{k},\left|y_{k}^{\prime}\right|\right) d t, \quad B=\int_{0}^{s}\left(\frac{t}{s}\right)^{N-1} F_{1}\left(t, y,\left|y^{\prime}\right|\right) d t .
$$

Note that $\Psi_{k}(s)=2^{1 /(p-1)} \int_{0}^{s}\left|F_{1}\left(t, y_{k},\left|y_{k}^{\prime}\right|\right)-F_{1}\left(t, y,\left|y^{\prime}\right|\right)\right| d t\left(A^{(2-p) /(p-1)}+B^{(2-p) /(p-1)}\right)$ satisfies

$$
\Psi_{k}(s) \leq 2^{(p+1) /(p-1)} \int_{0}^{s} F_{1}^{1 /(p-1)}\left(t, \eta, \frac{m \eta}{p}\right) d t
$$

$\Psi_{k}(s) \rightarrow 0$ pointwise on $[0, \infty)$ as $k \rightarrow \infty$. From the Lebesgue dominated convergence theorem, it follows that $F y_{k}(r)$ converges to $F y(r)$ uniformly on $[0, \infty)$ as $k \rightarrow \infty$, and hence $F y_{k}(r) \rightarrow F y(r)$ in $C[0, \infty)$ as $k \rightarrow \infty$. On the other hand,

$$
\begin{aligned}
& \left|\left(F y_{k}\right)^{\prime}(r)-(F y)^{\prime}(r)\right| \\
& \quad=\left|\Phi_{p}^{-1}\left(\int_{0}^{s}\left(\frac{t}{s}\right)^{N-1} F_{1}\left(t, y_{k},\left|y_{k}^{\prime}\right|\right) d t\right)-\Phi_{p}^{-1}\left(\int_{0}^{s}\left(\frac{t}{s}\right)^{N-1} F_{1}\left(t, y,\left|y^{\prime}\right|\right) d t\right)\right| .
\end{aligned}
$$

Similarly, we have $\left(F y_{k}\right)^{\prime}(r)$ converging to $(F y)^{\prime}(r)$ uniformly on $[0, \infty)$ as $k \rightarrow \infty$. Thus, $F$ is continuous.

Proposition 3.4. FY is relatively compact.

It suffices to show that $\{F y(r) \mid y \in Y\}$ and $\left\{(F y)^{\prime}(r) \mid y \in Y\right\}$ are uniformly bounded and equicontinuous on every subset $[0, M] \subset[0, \infty)$. From (3.20), (3.21), the uniform boundedness is obvious, so we only prove that $\left\{(F y)^{\prime}(r) \mid y \in Y\right\}$ is equicontinuous in $[0, M]$.

From (3.21), we obtain

$$
\begin{gathered}
(F y)^{\prime}(r)=\left(\int_{0}^{r}\left(\frac{s}{r}\right)^{N-1} F_{1}\left(s, y(s),\left|y^{\prime}(s)\right|\right) d s\right)^{1 /(p-1)}, \\
\left(\Phi_{p}\left((F y)^{\prime}(r)\right)\right)^{\prime}=F_{1}\left(r, y(r),\left|y^{\prime}(r)\right|\right)-(N-1) \int_{0}^{r} s^{N-1} r^{-N} F_{1}\left(s, y,\left|y^{\prime}(s)\right|\right) d s
\end{gathered}
$$


then

$$
\left|\left(\Phi_{p}\left((F y)^{\prime}(r)\right)\right)^{\prime}\right| \leq F_{1}\left(r, \eta, \frac{m \eta}{p}\right)+\frac{N-1}{r} \int_{0}^{r} F_{1}\left(s, \eta, \frac{m \eta}{p}\right) d s .
$$

From (3.30), we have

$$
\lim _{r \rightarrow 0^{+}}\left(\Phi_{p}\left((F y)^{\prime}(r)\right)\right)^{\prime}=\frac{1}{N} F_{1}\left(0, y(0),\left|y^{\prime}(0)\right|\right),
$$

then

$$
\left(\Phi_{p}(F y)^{\prime}\right)^{\prime}(0)=\lim _{r \rightarrow 0^{+}} \frac{\left[\Phi_{p}\left((F y)^{\prime}(r)\right)-\Phi_{p}\left((F y)^{\prime}(0)\right)\right]}{r}=\frac{1}{N} F_{1}\left(0, y(0),\left|y^{\prime}(0)\right|\right) .
$$

Therefore,

$$
\left|\left(\Phi_{p}\left((F y)^{\prime}\right)\right)^{\prime}(0)\right|=\frac{1}{N} F_{1}\left(0, y(0),\left|y^{\prime}(0)\right|\right) \leq F_{1}\left(0, \eta, \frac{m \eta}{p}\right) .
$$

For (3.25), we let

$$
\left.\left[\frac{N-1}{r} \int_{0}^{r} F_{1}\left(s, \eta, \frac{m \eta}{p}\right) d s\right]\right|_{r=0}=(N-1) F_{1}\left(0, \eta, \frac{m \eta}{p}\right),
$$

and then (3.31) holds for all $r \geq 0$, therefore

$$
\mid\left(\Phi_{p}\left((F y)^{\prime}\right)^{\prime}(r) \mid \leq F_{1}\left(r, \eta, \frac{m \eta}{p}\right)+\frac{N-1}{r} \int_{0}^{r} \frac{F_{1}(s, \ldots)}{r} d s, \quad r \geq 0 .\right.
$$

Consequently, for $\left((F y)^{\prime}(r)\right)^{\prime}$ we have estimates in $[0, M]$ :

$$
\begin{aligned}
\max _{0 \leq r \leq M} & \left|\Phi_{p}\left((F y)^{\prime}(r)\right)^{\prime}\right| \\
& \leq \max _{0 \leq r \leq M} F_{1}\left(r, \eta, \frac{m \eta}{p}\right)+\max _{0 \leq r \leq M}\left|\frac{N-1}{r} \int_{0}^{r} F_{1}\left(s, \eta, \frac{m \eta}{p}\right) d s\right|=L_{M},
\end{aligned}
$$

then

$$
\left|\Phi_{p}\left((F y)^{\prime}\left(r_{1}\right)\right)-\Phi_{p}\left((F y)^{\prime}\left(r_{2}\right)\right)\right| \leq L_{M}\left|r_{1}-r_{2}\right|, \quad r_{1}, r_{2} \in[0, M] .
$$

On the other hand,

$$
\left|(F y)^{\prime}\left(r_{1}\right)-(F y)^{\prime}\left(r_{2}\right)\right| \leq \sup \left|\left(\Phi_{p}^{-1}\right)^{\prime}(x)\right|\left|r_{1}-r_{2}\right|, \quad r_{1}, r_{2} \in[0, M],
$$

then $\left\{(F y)^{\prime}(r) \mid y \in Y\right\}$ is equicontinuous in $[0, M]$.

To prove that $F Y$ is relatively compact in $Y$, we will prove every sequence $\left\{F y_{k}(r)\right\}$ which has convergent subsequence in $Y$. We only need to use Ascoli-Arzela theorem in turn for the sequence of interval $\left[0, M_{1}\right] \subset\left[0, M_{2}\right] \subset \cdots \subset\left[0, M_{j}\right] \subset \cdots$ (where $M_{j} \rightarrow \infty$ as $j \rightarrow \infty$ ), and use the diagonalization argument as in [25]. Thus we are able to apply the 
Schauder-Tychonoff fixed point theorem and conclude that $F$ has a fixed point $y$ in $Y$. This fixed point $y=y(r)$ is a solution of (3.9), and so we obtain a supersolution $y(x)$ of (1.1) in $\mathbb{R}^{N}$ defined by $y(x)=y(|x|)$.

(ii) For $2<p \leq N+1$, since $\Phi_{p}^{-1} \notin C^{1}(R)$ ( $\Phi_{p}^{-1}$ is the inverse function of $\Phi_{p}(y)=$ $\left.|y|^{p-2} y\right)$, then we consider the perturbation equation

$$
\begin{gathered}
\left(r^{N-1} g_{\epsilon}\left(y^{\prime}\right)\right)^{\prime}=r^{N-1} F_{1}\left(r, y,\left|y^{\prime}\right|\right), \\
y(0)=\eta, \quad y^{\prime}(0)=0,
\end{gathered}
$$

where $g_{\epsilon}(y)=\epsilon y+\Phi_{p}(y)$. From $g_{\epsilon}^{-1} \in C^{1}(R)$, and then similarly with (i), we have for all $\epsilon>0$, (3.40), (3.41) has a bounded positive solution $y_{\epsilon} \in C^{1}(R)$, and similarly with (i), we have

$$
\left.\max _{0 \leq r \leq M}\left|\left(g_{\epsilon}\left(\left(F y_{\epsilon}\right)^{\prime}(r)\right)\right)^{\prime}(r)\right| \leq L_{M} \quad \text { (be independent of } \epsilon\right)
$$

From Ascoli-Arzela theorem, we have $g_{\epsilon}\left(y_{\epsilon}^{\prime}\right) \rightarrow v(\epsilon \rightarrow 0)$, where $v \in C(R)$. Since $\left\|y_{\epsilon}^{\prime}\right\|_{0}$ is bounded, it follows that $\Phi_{p}\left(y_{\epsilon}\right) \rightarrow v(\epsilon \rightarrow 0)$. Since $\Phi_{p}$ is $R \rightarrow R$ strictly increasing which implies that $y_{\epsilon}^{\prime} \rightarrow \Phi_{p}^{-1}(v)(\epsilon \rightarrow 0)$, then we have

$$
y_{\epsilon}=\eta+\int_{0}^{r} y_{\epsilon}^{\prime}(s) d s \longrightarrow \eta+\int_{0}^{t} \Phi_{p}^{-1}(v(s)) d s=y \quad(\epsilon \longrightarrow 0) .
$$

From (3.40), (3.41), we have $y_{\epsilon}$ satisfying

$$
\begin{gathered}
\epsilon r^{N-1} y_{\epsilon}^{\prime}+r^{N-1} \Phi_{p}\left(y_{\epsilon}^{\prime}\right)=\eta+\int_{0}^{s} s^{N-1} F_{1}\left(s, y_{\epsilon}, y_{\epsilon}^{\prime}\right) d s \\
y_{\epsilon}(0)=\eta, \quad y_{\epsilon}^{\prime}(0)=0
\end{gathered}
$$

and then for all $r \in[0, M]$, let $\epsilon \rightarrow 0, y \in C^{1}[0, M]$ a bounded entire solution of (3.7).

Similarly, we consider the initial value problem for (3.8):

$$
\begin{gathered}
\left(\Phi_{p}\left(z^{\prime}\right)\right)^{\prime}+\frac{N-1}{r} \Phi_{p}\left(z^{\prime}\right)=F_{2}\left(r, z,\left|z^{\prime}\right|\right), \quad r>0, \\
z(0)=\xi, \quad z^{\prime}(0)=0,
\end{gathered}
$$

where $\xi$ is a real number which is determined below. Further we consider equivalence integral equation equivalent to (3.45):

$$
z(r)=\xi+\int_{0}^{r}\left(\int_{0}^{s}\left(\frac{t}{s}\right)^{N-1} F_{2}\left(t, z,\left|z^{\prime}\right|\right) d t\right)^{1 /(p-1)} d s
$$

From (3.14), we can choose sufficiently large constant $\xi>0$, satisfying

$$
\int_{0}^{\infty} K(s) F_{2}^{1 /(p-1)}\left(s, \xi, \frac{m \xi}{p}\right) d s<\frac{\xi}{p} .
$$


Let us set

$$
Z=\left\{z \in C^{1}[0, \infty) \mid \xi \leq z(r) \leq 2 \xi, 0 \leq z^{\prime}(r) \leq \frac{m \xi}{p}, r \geq 0\right\}
$$

Define mapping $\Psi$ :

$$
\Psi(r)=\xi+\int_{0}^{r}\left(\int_{0}^{s}\left(\frac{t}{s}\right)^{N-1} F_{2}\left(t, z,\left|z^{\prime}\right|\right) d t\right)^{1 /(p-1)} d s
$$

Similarly, $\Psi(r)$ has a fixed point in $z \in Z$.

In the above, constants $\eta$ and $\xi$ have to satisfy not only (3.14) and (3.40), but also they need to satisfy $2 \xi \leq \eta$ (this we can realize choosing first $\xi$ which satisfies (3.40), fixing, and finally adjusting $\xi$, so that we choose $\eta$ which satisfies $2 \xi \leq \eta$ and (3.18)). Therewith, we have

$$
0<\xi \leq z(r) \leq 2 \xi \leq \eta \leq y(r) \leq 2 \eta
$$

On the other hand, if the problem (3.9) has a solution $y(r)$, then $v(x)=y(|x|)=y(r)$ is a solution of (3.7). Similarly, if the initial value problem (3.45) has solutions $z(r)$, then $w(x)=z(|x|)=z(r)$ is a solution of (3.8). From (3.50), it follows that

$$
0<\xi \leq w(x) \leq v(x) \leq 2 \eta, \quad x \in \mathbb{R}^{N} .
$$

From Lemma 2.2, (1.1) has at least a solution $u(x)$ and satisfies

$$
w(x) \leq u(x) \leq v(x), \quad x \in \mathbb{R}^{N} .
$$

Here and now we have proved that (1.1) exists for positive entire solution $u(x)$. From (3.51), (3.52), we see that all super- and subbounds of positive solutions are dependent choice of sufficiently large positive number $\xi$, $\eta$. If we choose the number pair $\left(\xi_{j}, \eta_{j}\right)$ $(j=1,2, \ldots)$, using the closed interval of the closed interval set which is each other nonintersect $\left\{\left(\xi_{j}, \eta_{j}\right) \mid j=1,2, \ldots\right\}$, then we obtain each other difference bounded positive solutions of $(1.1) u_{j}(x), j=1,2, \ldots$, therefore (1.1) possesses infinitely many positive entire solutions.

Remark 3.5. When $p=2$, in $[11,20,21]$ relative results were obtained and our results can be seen as their extensions.

Remark 3.6. If condition (II) of Theorem 3.1 is replaced by

(II) $)_{2}$ (a) for $1<p<2$ and fixed $r \in \mathbb{R}^{+}, F_{2}^{1 /(p-1)}(r, \lambda, m \lambda / p) / \lambda$ is nondecreasing for $\lambda \in$ $(0, \infty)$ and satisfies

$$
\lim _{\lambda \rightarrow 0^{+}} \frac{F_{2}^{1 /(p-1)}(r, \lambda, m \lambda / p)}{\lambda}=0,
$$

where $m=((2-p) /(N-p+1))^{(2-p) /(p-1)}$; 
(b) for $2 \leq p \leq N+1$ and fixed $r \in \mathbb{R}^{+}, F_{2}^{p-1}\left(r, \lambda, m_{1} \lambda / p\right) / \lambda$ is nondecreasing for $\lambda \in(0, \infty)$ and satisfies

$$
\lim _{\lambda \rightarrow 0^{+}} \frac{F_{2}^{p-1}\left(r, \lambda, m_{1} \lambda / p\right)}{\lambda}=0
$$

where $m_{1}=((p-2) /(N(p-1)-1))^{(p-2) /(p-1)^{2}}$;

then the conclusion of Theorem 3.1 holds.

Theorem 3.7. Suppose that $f$ satisfies $(A)-(C)$ and the following conditions hold.

(I) $F_{1}(r, u, v)$ and $F_{2}(r, u, v)$ are nondecreasing for $u \in \mathbb{R}^{+}$, and nonincreasing for $v \in$ $\mathbb{R}^{+}$.

(II) (a) For $1<p<2$ and fixed $r \in \mathbb{R}^{+}, F_{2}^{1 /(p-1)}(r, \lambda, 0) / \lambda$ is nonincreasing for $\lambda \in(0, \infty)$ and satisfies

$$
\lim _{\lambda \rightarrow \infty} \frac{F_{2}^{1 /(p-1)}(r, \lambda, 0)}{\lambda}=0 .
$$

(b) For $2 \leq p \leq N+1$ and fixed $r \in \mathbb{R}^{+}, F_{2}^{p-1}(r, \lambda, 0) / \lambda$ is nonincreasing for $\lambda \in$ $(0, \infty)$ and satisfies

$$
\lim _{\lambda \rightarrow \infty} \frac{F_{2}^{p-1}(r, \lambda, 0)}{\lambda}=0 .
$$

(III) (a) For $1<p<2$, there exists a positive constant $c$ such that

$$
\int_{0}^{\infty} K(s) F_{2}^{1 /(p-1)}(s, c, 0) d s<\infty
$$

where

$$
K(s)= \begin{cases}1 & \text { if } 0 \leq s<1 \\ s^{1 /(p-1)} & \text { if } s>1\end{cases}
$$

(b) For $2 \leq p \leq N+1$, there exists a positive constant c such that

$$
\int_{0}^{\infty} K(s) F_{2}^{p-1}(s, c, 0) d s<\infty,
$$

where

$$
K(s)= \begin{cases}1 & \text { if } 0 \leq s<1, \\ s^{p-1} & \text { if } s>1 .\end{cases}
$$

Then the conclusion of Theorem 3.1 holds. 
14 Multiple entire solutions for quasilinear elliptic equations

Remark 3.8. If condition (II) of Theorem 3.7 is replaced by

$(\mathrm{II})_{2}$ (a) for $1<p<2$ and fixed $r \in \mathbb{R}^{+}, F_{2}^{1 /(p-1)}(r, \lambda, 0) / \lambda$ is nondecreasing for $\lambda \in$ $(0, \infty)$ and satisfies

$$
\lim _{\lambda \rightarrow 0^{+}} \frac{F_{2}^{1 /(p-1)}(r, \lambda, 0)}{\lambda}=0 ;
$$

(b) for $2 \leq p \leq N+1$ and fixed $r \in \mathbb{R}_{+}, F_{2}^{p-1}(r, \lambda, 0) / \lambda$ is nondecreasing for $\lambda \in$ $(0, \infty)$ and satisfies

$$
\lim _{\lambda \rightarrow 0^{+}} \frac{F_{2}^{p-1}(r, \lambda, 0)}{\lambda}=0 ;
$$

then the conclusion of Theorem 3.1 holds.

Theorem 3.9. Assume that $f$ satisfies $(A)-(C)$ and the following conditions hold.

(I) $F_{1}(r, u, v)$ and $F_{2}(r, u, v)$ are nonincreasing in $u \in(0, \infty)$ and nonincreasing in $v \in$ $(0, \infty)$ for all fixed $r \in \mathbb{R}^{+}$.

(II) (a) For $1<p<2$, there exists a positive constant $c$ such that

$$
\int_{0}^{\infty} K(s) F_{2}^{1 /(p-1)}(s, c, 0) d s<\infty
$$

where

$$
K(s)= \begin{cases}1 & \text { if } 0 \leq s<1 \\ s^{1 /(p-1)} & \text { if } s>1\end{cases}
$$

(b) For $2 \leq p \leq N+1$, there exists a positive constant c such that

$$
\int_{0}^{\infty} K(s) F_{2}^{p-1}(s, c, 0) d s<\infty
$$

where

$$
K(s)= \begin{cases}1 & \text { if } 0 \leq s<1 \\ s^{p-1} & \text { if } s>1 .\end{cases}
$$

Then the conclusion of Theorem 3.1 holds.

Theorem 3.10. Assume that $f$ satisfies $(A)-(C)$ and the following conditions hold.

(I) $F_{1}(r, u, v)$ and $F_{2}(r, u, v)$ are nondecreasing in $u \in(0, \infty)$ and nondecreasing in $v \in$ $(0, \infty)$ for all fixed $r \in \mathbb{R}^{+}$. 
(II) (a) For $1<p<2$ and fixed $r \in \mathbb{R}^{+}, F_{2}^{1 /(p-1)}(r, p \lambda, m \lambda / p) / \lambda$ is nonincreasing for $\lambda \in$ $(0, \infty)$ and satisfies

$$
\lim _{\lambda \rightarrow+\infty} \frac{F_{2}^{1 /(p-1)}(r, p \lambda, m \lambda / p)}{\lambda}=0,
$$

where $m=((2-p) /(N-p+1))^{(2-p) /(p-1)}$.

(b) For $2 \leq p \leq N+1$ and fixed $r \in \mathbb{R}^{+}, F_{2}^{p-1}\left(r, p \lambda, m_{1} \lambda / p\right) / \lambda$ is nonincreasing for $\lambda \in(0, \infty)$ and satisfies

$$
\lim _{\lambda \rightarrow+\infty} \frac{F_{2}^{p-1}\left(r, p \lambda, m_{1} \lambda / p\right)}{\lambda}=0,
$$

where $m_{1}=((p-2) /(N(p-1)-1))^{(p-2) /(p-1)^{2}}$.

(III) (a) For $1<p<2$, there exists a positive constant $c>0$ such that

$$
\int_{0}^{\infty} K(s) F_{2}^{1 /(p-1)}\left(s, p c, \frac{m c}{p}\right) d s<\infty
$$

where

$$
K(s)= \begin{cases}1 & \text { if } 0 \leq s<1 \\ s^{1 /(p-1)} & \text { if } s>1\end{cases}
$$

(b) For $2 \leq p \leq N+1$, there exists a positive constant $c$ such that

$$
\int_{0}^{\infty} K(s) F_{2}^{p-1}\left(s, p c, \frac{m_{1} c}{p}\right) d s<\infty
$$

where

$$
K(s)= \begin{cases}1 & \text { if } 0 \leq s<1, \\ s^{p-1} & \text { if } s>1 .\end{cases}
$$

Then the conclusion of Theorem 3.1 holds.

\section{Example}

Example 4.1. Consider the equation

$$
\operatorname{div}\left(|\nabla u|^{p-2} \nabla u\right)=\psi(x) e^{-|x|^{2} u^{\alpha}(p-1)}|\nabla u|^{p-1}, \quad x \in \mathbb{R}^{N}, N \geq 3,
$$

where $0<\alpha<p-1, p>1$, and $\psi(x)>0$ is locally Lipschitz continuous in $\mathbb{R}^{N}$. 
16 Multiple entire solutions for quasilinear elliptic equations

Let $f(x, u, \nabla u)=\psi(x) e^{-|x|^{2} u^{\alpha}(p-1)}|\nabla u|^{p-1}, \quad \psi^{*}(r)=\max _{|x|=r} \psi(x)$, and $\psi_{*}(r)=$ $\min _{|x|=r} \psi(x)$. Choose

$$
\begin{aligned}
& F_{1}(|x|, u,|v|)= \begin{cases}\psi_{*}(|x|) e^{-|x|^{2} u^{\alpha}(p-1)}|v|^{p-1} & \text { if } 1<p<2, \\
\psi_{*}(|x|) e^{-|x|^{2} u^{\alpha} /(p-1)}|v|^{1 /(p-1)} & \text { if } p \geq 2,\end{cases} \\
& F_{2}(|x|, u,|v|)= \begin{cases}\psi^{*}(|x|) e^{-|x|^{2} u^{\alpha}(p-1)}|v|^{p-1} & \text { if } 1<p<2, \\
\psi^{*}(|x|) e^{-|x|^{2} u^{\alpha} /(p-1)}|v|^{1 /(p-1)} & \text { if } p \geq 2 .\end{cases}
\end{aligned}
$$

It is easy to check that (i) $F_{1}(r, u, v), F_{2}(r, u, v)$ satisfy condition (I) of Theorem 3.1; (ii) $F_{2}(r, u, v)$ satisfies condition (II) of Theorem 3.1, $F_{1}(r, u, t), F_{2}(r, u, t)$ are nonincreasing functions for $u \in \mathbb{R}^{+}$, which is a nondecreasing function for $t \in \mathbb{R}^{+}$, and for $1<p<2$ and fixed $r \in \mathbb{R}^{+}$,

$$
\frac{F_{2}^{1 /(p-1)}(r, \lambda, m \lambda / p)}{\lambda}=\left(\psi^{*}(r)\right)^{1 /(p-1)} e^{-r^{2} \lambda^{\alpha}} \frac{p}{m}
$$

is nonincreasing for $\lambda \in(0, \infty)$ and satisfies

$$
\lim _{\lambda \rightarrow+\infty} \frac{F_{2}^{p-1}(r, \lambda, m \lambda / p)}{\lambda}=0
$$

where $m=((2-p) /(N-p+1))^{(2-p) /(p-1)}$. For $p \geq 2$, being similar to $1<p<2$, suppose that

$$
\int_{0}^{\infty} K(s) F_{2}^{1 /(p-1)}\left(s, c, \frac{m c}{p}\right) d s=\left(\frac{m c}{p}\right)^{p-1} \int_{0}^{\infty} K(s)\left(\psi^{*}(s)\right)^{1 /(p-1)} e^{-s^{2} c^{\alpha}} d s<\infty
$$

(where $c$ is a certain positive constant) holds. Then from Theorem 3.1 it follows that (4.1) possesses infinitely many positive entire solutions $u(x)$.

Example 4.2. Consider the equation

$$
\operatorname{div}\left(|\nabla u|^{p-2} \nabla u\right)=\psi(x) u^{\alpha(p-1)} e^{-|x||\nabla u|^{\beta}(p-1)}, \quad x \in \mathbb{R}^{N}
$$

where $\alpha, \beta \in(0, p-1), p>1$.

Let

$$
\begin{gathered}
f(x, u, \nabla u)=\psi(x) u^{\alpha(p-1)} e^{-|x||\nabla u|^{\beta}(p-1)}, \\
F_{1}(|x|, u,|v|)= \begin{cases}\psi_{*}(|x|) u^{\alpha(p-1)} e^{-|x|^{2}|v|^{\beta}(p-1)} & \text { if } 1<p<2, \\
\psi_{*}(|x|) u^{\alpha /(p-1)} e^{-|x|^{2}|v|^{\beta} /(p-1)} & \text { if } p \geq 2,\end{cases} \\
F_{1}(|x|, u,|v|)= \begin{cases}\psi^{*}(|x|) u^{\alpha(p-1)} e^{-|x|^{2}|v|^{\beta}(p-1)} & \text { if } 1<p<2, \\
\psi^{*}(|x|) u^{\alpha /(p-1)} e^{-|x|^{2}|v|^{\beta /(p-1)}} & \text { if } p \geq 2,\end{cases}
\end{gathered}
$$


where $\psi(x), \psi_{*}(r), \psi^{*}(r)$ are defined in Example 4.1. It is easy to prove that the conditions of Theorem 3.1 are satisfied. Suppose that

$$
\int_{0}^{\infty} K(s)\left(\psi^{*}(s)\right)^{1 /(p-1)} d s<\infty
$$

holds. Then (4.6) possesses infinitely many positive entire solutions $u(x)$.

Example 4.3. Consider the equation

$$
\operatorname{div}\left(|\nabla u|^{p-2} \nabla u\right)=\psi(x)\left(u^{\alpha}+u^{\beta}+|\nabla u|^{\gamma}\right), \quad x \in \mathbb{R}^{N}, N \geq 3
$$

where $\psi(x), \psi_{*}(r), \psi^{*}(r)$ are defined in Example 4.1, $\alpha, \beta, \gamma \in(0,1)$, and $\beta+\gamma<p-1$, $p>1$. It is easy to prove that the conditions of Theorem 3.7 are satisfied. Moreover, suppose that

$$
\int_{0}^{\infty} K(s)\left(\psi^{*}(s)\right)^{1 /(p-1)} d s<\infty
$$

holds. Then (4.9) possesses infinitely many positive entire solutions $u(x)$.

Example 4.4. Consider the equation

$$
\operatorname{div}\left(|\nabla u|^{p-2} \nabla u\right)=\psi(x) \frac{|\nabla u|^{\beta}}{(1+u)^{\alpha}},
$$

where $\alpha, \beta>0$ and $\beta-\alpha>p-1, p>1$, with $\psi(x), \psi_{*}(r), \psi^{*}(r)$ are defined in Example 4.1. It is easy to prove that the conditions of Theorem 3.9 are satisfied. Moreover, suppose that

$$
\int_{0}^{\infty} K(s)\left(\psi^{*}(s)\right)^{1 /(p-1)} d s<\infty
$$

holds. Then (4.11) possesses infinitely many positive entire solutions $u(x)$.

Example 4.5. Consider the equation

$$
\triangle u=e^{-\gamma|x|} u^{\alpha}|\nabla u|^{\beta}, \quad x \in \mathbb{R}^{2},
$$

where $\alpha, \beta, \gamma$ are all positive constants. Let $f(r, u, v)=e^{\gamma r} u^{\alpha} v^{\beta}$. Clearly if $\gamma>0, \beta \geq$ 0 , either $\alpha+\beta<p-1$ or $\alpha+\beta>p-1$, then it is easy to verify that the conditions of Theorem 3.10 are satisfied. Thus, Theorem 3.1 holds for (4.13).

\section{Acknowledgments}

This project was supported by the National Natural Science Foundation of China (No. 10571022), the Natural Science Foundation of Educational Department of Jiangsu Province (No. 04KJB110062), and the Science Foundation of Nanjing Normal University (No. 2003SXXXGQ2B37). 


\section{References}

[1] H. Amann, Fixed point equations and nonlinear eigenvalue problems in ordered Banach spaces, SIAM Review 18 (1976), no. 4, 620-709.

[2] J. R. Esteban and J. L. Vázquez, On the equation of turbulent filtration in one-dimensional porous media, Nonlinear Analysis 10 (1986), no. 11, 1303-1325.

[3] B. Gidas, W. M. Ni, and L. Nirenberg, Symmetry and related properties via the maximum principle, Communications in Mathematical Physics 68 (1979), no. 3, 209-243.

[4] M. Guedda and L. Véron, Local and global properties of solutions of quasilinear elliptic equations, Journal of Differential Equations 76 (1988), no. 1, 159-189.

[5] Z. M. Guo, Some existence and multiplicity results for a class of quasilinear elliptic eigenvalue problems, Nonlinear Analysis 18 (1992), no. 10, 957-971.

[6] Z. M. Guo and J. R. L. Webb, Uniqueness of positive solutions for quasilinear elliptic equations when a parameter is large, Proceedings of the Royal Society of Edinburgh. Section A 124 (1994), no. $1,189-198$.

[7] M. A. Herrero and J. L. Vázquez, On the propagation properties of a nonlinear degenerate parabolic equation, Communications in Partial Differential Equations 7 (1982), no. 12, 1381-1402.

[8] S. Kamin and L. Véron, Flat core properties associated to the p-Laplace operator, Proceedings of the American Mathematical Society 118 (1993), no. 4, 1079-1085.

[9] S. Kichenassamy and J. Smoller, On the existence of radial solutions of quasi-linear elliptic equations, Nonlinearity 3 (1990), no. 3, 677-694.

[10] T. Kusano and C. A. Swanson, Positive entire solutions of semilinear biharmonic equations, Hiroshima Mathematical Journal 17 (1987), no. 1, 13-28.

[11] T. Kusano and H. Usami, Positive solutions of a class of second order semilinear elliptic equations in the plane, Mathematische Annalen 268 (1984), no. 2, 255-264.

[12] O. A. Ladyzhenskaya and N. N. Ural'tseva, Linear and Quasilinear Elliptic Equations, Academic Press, New York, 1968.

[13] G. M. Lieberman, Boundary regularity for solutions of degenerate elliptic equations, Nonlinear Analysis 12 (1988), no. 11, 1203-1219.

[14] Q. Lu, Z. Yang, and E. H. Twizell, Existence of entire explosive positive solutions of quasi-linear elliptic equations, Applied Mathematics and Computation 148 (2004), no. 2, 359-372.

[15] M. Montenegro and M. Montenegro, Existence and nonexistence of solutions for quasilinear elliptic equations, Journal of Mathematical Analysis and Applications 245 (2000), no. 2, 303-316.

[16] C. V. Pao, Positive solutions of a nonlinear boundary-value problem of parabolic type, Journal of Differential Equations 22 (1976), no. 1, 145-163.

[17] S. I. Pohožaev, On equations of the form $\Delta u=f(x, u, \nabla u)$, Mathematics of the USSR. Sbornik 41 (1982), no. 2, 269-280.

[18] D. Ruiz, A priori estimates and existence of positive solutions for strongly nonlinear problems, Journal of Differential Equations 199 (2004), no. 1, 96-114.

[19] P. Tolksdorf, On the Dirichlet problem for quasilinear equations in domains with conical boundary points, Communications in Partial Differential Equations 8 (1983), no. 7, 773-817.

[20] X. Xu, Positive entire solutions to a class of nonlinear elliptic equations, Journal of Mathematics (Wuhan) 16 (1996), no. 4, 403-411 (Chinese).

[21] X. Xu, B. Yang, and L. Debnath, Positive entire solutions of nonlinear polyharmonic equations in $\mathbb{R}^{2}$, Applied Mathematics and Computation 126 (2002), no. 2-3, 377-388.

[22] Z. Yang, Non-existence of positive entire solutions for elliptic inequalities of p-Laplacian, Applied Mathematics. A Journal of Chinese Universities. Series B 12 (1997), no. 4, 399-410 (Chinese).

[23] _ Existence of entire explosive positive radial solutions of quasilinear elliptic systems, International Journal of Mathematics and Mathematical Sciences 2003 (2003), no. 46, 2907-2927.

[24] _ Existence of positive bounded entire solutions for quasilinear elliptic equations, Applied Mathematics and Computation 156 (2004), no. 3, 743-754. 
[25] _ Existence of positive entire solutions for singular and non-singular quasi-linear elliptic equation, to appear in Journal of Computational and Applied Mathematics, Corrected Proof, Available online 4 January 2006.

[26] Z. Yang and Z. Guo, On the structure of positive solutions for quasilinear ordinary differential equations, Applicable Analysis 58 (1995), no. 1-2, 31-52.

[27] Z. Yang and H. Yang, Existence of positive radial solutions for quasilinear elliptic boundary value problems, Annals of Differential Equations 15 (1999), no. 4, 438-452 (Chinese).

Yang Zuodong: School of Mathematics and Computer Science, Nanjing Normal University, Jiangsu, Nanjing 210097, China

E-mail address: zdyang_jin@263.net 


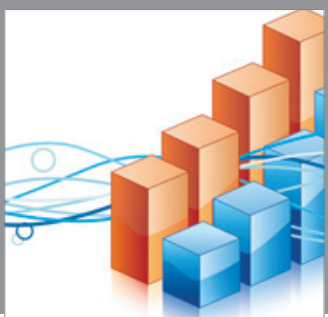

Advances in

Operations Research

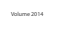

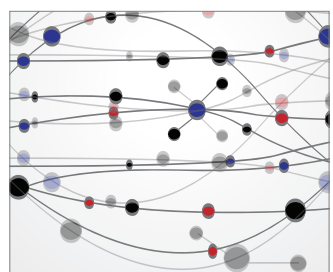

\section{The Scientific} World Journal
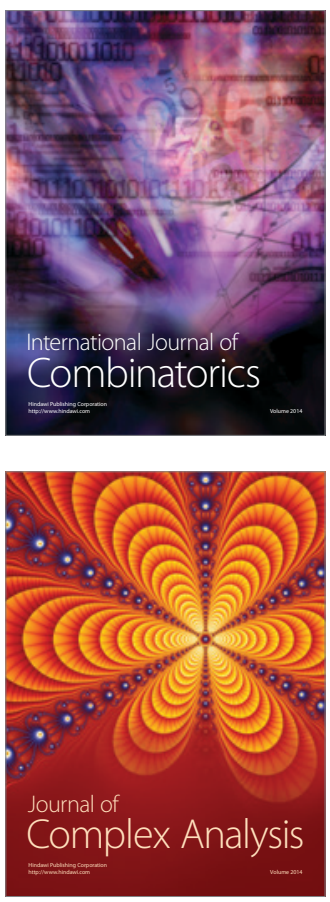

International Journal of

Mathematics and

Mathematical

Sciences
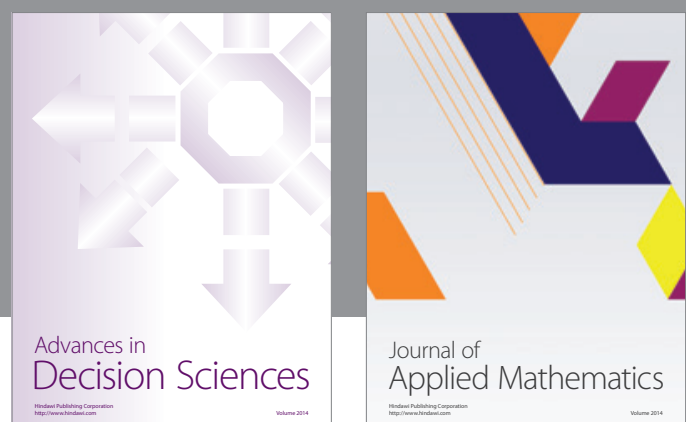

Journal of

Applied Mathematics
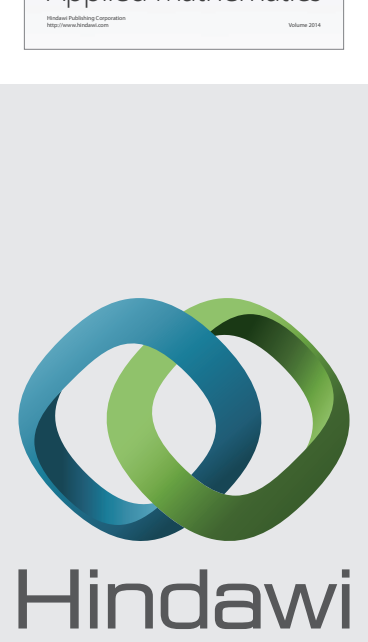

Submit your manuscripts at http://www.hindawi.com
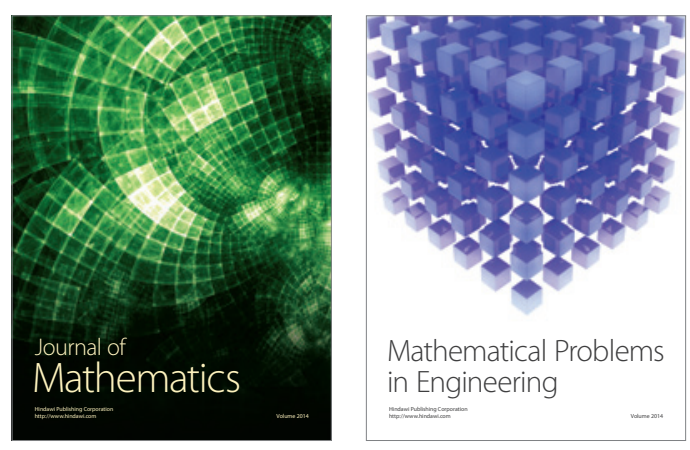

Mathematical Problems in Engineering
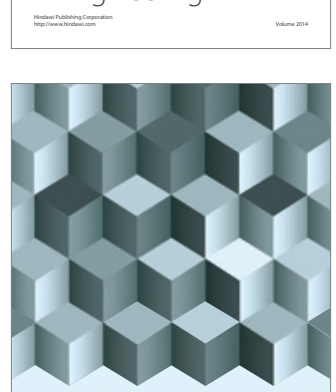

Journal of

Function Spaces
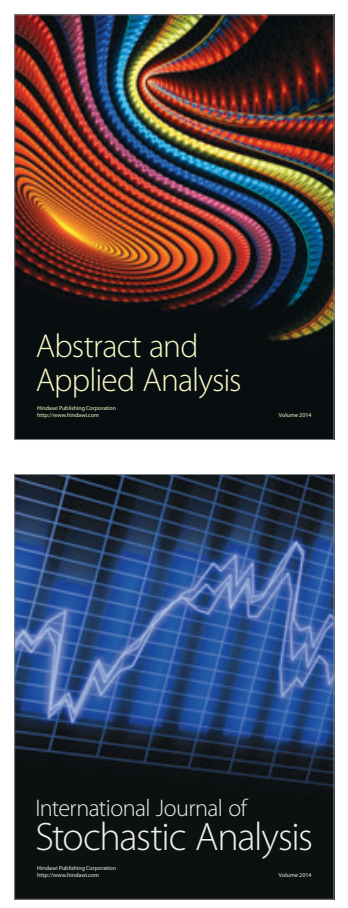

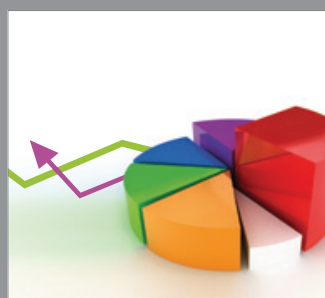

ournal of

Probability and Statistics

Promensencen
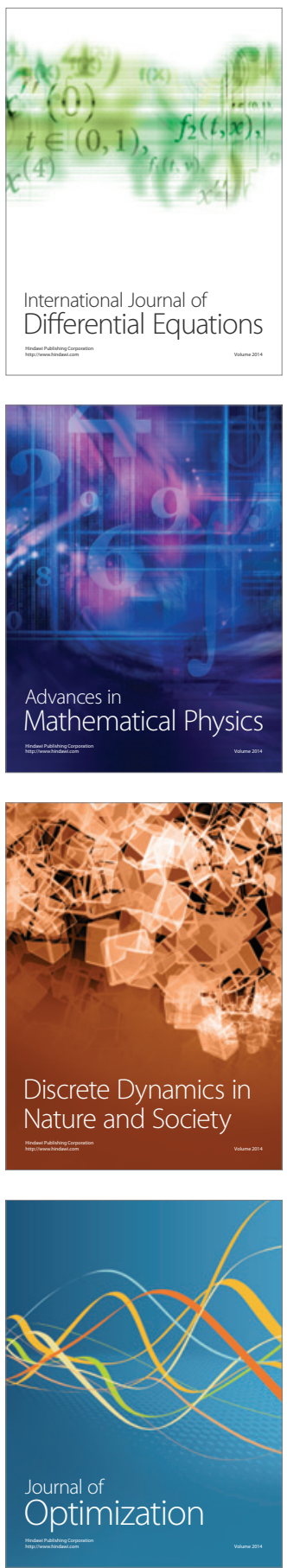